\title{
A catalog of human genes and a gene network controlling feeding behavior and body weight
}

\author{
E.V. Ignatieva \\ Institute of Cytology and Genetics SB RAS, Novosibirsk, Russia \\ Novosibirsk State University, Novosibirsk, Russia \\ e-mail: eignat@bionet.nsc.ru
}

Key words: feeding behaviour, body mass index, database, PPI network, obesity

Motivation and Aim: Obesity is a heritable disease with only a few safe and long-term effective therapies and intervention strategies. In efforts to understand the genetic basis of obesity and to collect data on potential targets for new therapies, we (1) created a catalog of human genes regulating feeding behavior (FB) or body weight (BW); (2) constructed protein-protein interaction (PPI) network involving genes/proteins from the catalog; (3) prioritized genes/proteins according to their degree in the PPI network.

Methods and Algorithms: The set of genes controlling FB or BW were collected from publications: (1) 578 human genes from the compendium of genes involved in regulation of FB or BW described in [1]; (2) 134 GWAS genes associated with elevated body mass index (BMI) revealed in [2]; (3) additional 190 GWAS genes associated with elevated BMI revealed in [3] at a less stringent significance level (between $5^{*} 10^{-8}$ and $10^{-5}$ ).

Results: We created a catalog comprising 853 human genes controlling FB and BW. According to the type of evidence, genes were classified into three functional categories: (1) GWAS genes from [1] and [2] (Sublist A); (2) other genes from [1] (Sublist B); (3) genes revealed in [3] at a less stringent significance level of $10^{-5}$ (Sublist C). Using STRING, GeneMANIA and Cytoscape, we constructed PPI network formed by genes/ proteins from the catalog and ranked all genes according to the number of neighbors in the network. The top genes/proteins were: (1) MAPK3, PPARG, and IRS1 in Sublist A; (2) ESR1, CREBBP, and STAT3 in Sublist B; (3) YWHAZ, NCK1, and MTOR in Sublist C. We revealed that six genes/proteins from the Sublist C (NCK1, ADCY5, PAM, NCAM1, $B A C E 2, M R A S$ ) had PPIs with genes/proteins associated with monogenic non-syndromic obesity.

Conclusion: By systematic review and curation of multiple lines of evidence, we created a comprehensive catalog of genes regulating FB and BW. Based on PPI network we found hubs among genes revealed by GWAS. We propose to keep in mind these genes as potential candidates for investigating the genetic factors predisposing to elevated body weight.

Acknowledgements: The work was supported by the Integrational Project of SB RAS No. 0324-2018-0021.

\section{References}

1. Ignatieva E.V. et al. (2016) A compendium of human genes regulating feeding behavior and body weight, its functional characterization and identification of GWAS genes involved in brain-specific PPI network. BMC Genetics. 17(Suppl 3):15.

2. Turcot V. et al. (2018) Protein-altering variants associated with body mass index implicate pathways that control energy intake and expenditure in obesity. Nat Genet. 50(1):26-41.

3. Locke A.E. et al. (2015) Genetic studies of body mass index yield new insights for obesity biology. Nature. 518:197-206. 